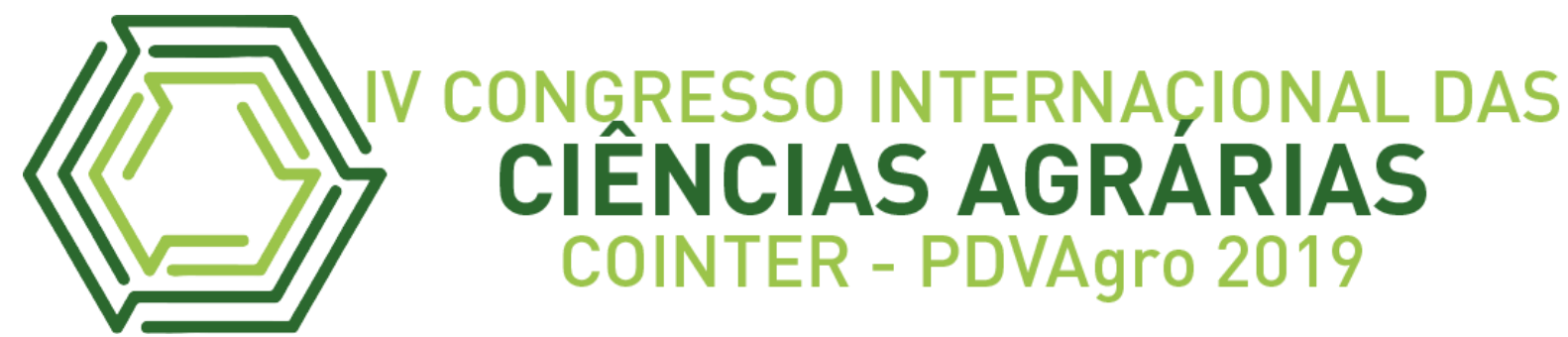

\title{
MÉTODOS DE INTERPRETAÇÃO PARA TESTE DE QUALIDADE EM SOLOS A PARTIR DA CROMATOGRAFIA CIRCULAR PLANA (FCC)
}

\section{MÉTODOS DE INTERPRETACIÓN PARA LA PRUEBA DE CALIDAD EN SOLES DE LA PLANA CIRCULAR CROMATOGRAFÍA (FCC)}

\section{INTERPRETATION METHODS FOR SOIL QUALITY TESTING FROM FLAT CIRCULAR CHROMATOGRAPHY (FCC)}

\author{
Apresentação: Comunicação Oral \\ Walter Santos Oliveira ${ }^{1}$; João Lucas do Vale Costa $^{2}$; Eldimar Pereira Cardoso ${ }^{3}$; Roseane de \\ Nazaré Pereira Teixeira ${ }^{4}$; Maria Eduarda da Silva Oliveira ${ }^{5}$
}

DOI: $\underline{\text { https://doi.org/10.31692/2526-7701.IVCOINTERPDVAgro.2019.0072 }}$

\begin{abstract}
Resumo
A cromatografia circular plana (FCC) é um método de separação de componentes que se divide em duas fases, fase estacionária e fase móvel. A fase estacionária envolve a reação de Hidróxido de Sódio $1 \%(\mathrm{NaOH})$ com amostra de solo e a impregnação de Nitrato de Prata $\left(\mathrm{AgNO}_{3}\right)$ no papel filtro. A fase móvel envolve a absorção pelo papel filtro da solução de Hidróxido de Sódio com solo. Atualmente a FCC indica uma leitura da qualidade da vida do solo através da harmonia de cores e desenhos entre os componentes minerais, orgânicos, energéticos e eletromagnéticos. Essa inter-relação compõe o metabolismo do solo vivo assim como a disponibilidade e eficiência, sendo, a solubilidade, concentração, constância e qualidade biológica dos nutrientes. No Brasil o método de análise tem sido amplamente divulgado nas ciências agrárias. No entanto, o desafio em interpretar o amplo espectro de resultados dos cromatogramas deu base para a escrita deste artigo cujo objetivo foi realizar uma sistematização das principais metodologias de análise de resultados e interpretações das zonas formadas por cromatogramas bem como de pesquisas inovadoras fora e dentro do Brasil. Os resultados apresentam pesquisas com a caracterização de tipos de manejo e arranjos produtivos de diversos de solos. Também, com o uso de softwares analisam propriedades de imagens, distribuição e números de partículas para a determinação de graus de tonalidade, saturação e entropia de cores. E, através de estatística experimental a correlação desses padrões cromatográficos e teores minerais e orgânicos de resultados de análise química de solos. Conclui-se que o avanço nas metodologias de compreensão dos resultados da FCC
\end{abstract}

\footnotetext{
${ }_{1}^{1}$ Agronomia, Instituto Federal de Educação, Ciência e Tecnologia do Pará, walterss@hotmail.com.br

${ }^{2}$ Agronomia, Instituto Federal de Educação, Ciência e Tecnologia do Pará, jlucasvalle1996@gmail.com

${ }^{3}$ Agronomia, Instituto Federal de Educação, Ciência e Tecnologia do Pará, eldcampones@gmail.com

${ }^{4}$ Agronomia, Instituto Federal de Educação, Ciência e Tecnologia do Pará, r.e.a.nazare.sph@gmail.com

${ }^{5}$ Profa Msc, Instituto Federal de Educação, Ciência e Tecnologia do Pará, profeduardasilva@gmail.com
} 
apresentou expressivo crescimento na última década, com o uso de métodos científicos e tecnológicos de alta precisão, abrindo a possibilidade de consolidar o método FCC como real instrumento de análise da saúde do solo assim como também de compostos. O presente artigo se apresenta como leitura para a prática de novas metodologias que levam a maior compreensão dos cromatogramas.

Palavras-Chave: Inovação social, saúde do solo, produção orgânica.

\section{Resumen}

La cromatografía circular plana (FCC) es un método de separación de componentes que se divide en dos fases, fase estacionaria y fase móvil. La fase estacionaria implica la reacción de hidróxido de sodio al $1 \%(\mathrm{NaOH})$ con la muestra del suelo y la impregnación de nitrato de plata $\left(\mathrm{AgNO}_{3}\right)$ en el papel filtrante. La fase móvil implica la absorción por el papel filtrante de la solución de hidróxido de sodio con el suelo. Actualmente, la cromatografía circular plana (FCC) indica una lectura de la calidad de la vida del suelo a través de la armonía de colores y diseños entre los componentes mineral, orgánico, energético y electromagnético. Esta interrelación constituye el metabolismo del suelo vivo, así como la disponibilidad y eficiencia, siendo la solubilidad, concentración, constancia y calidad biológica de los nutrientes. En Brasil, el método de análisis ha sido ampliamente difundido en las ciencias agrarias. Sin embargo, el reto de interpretar el amplio espectro de resultados de cromatogramas se basó en la redacción de este artículo, que tenía como objetivo realizar una sistematización de las principales metodologías de análisis de resultados e interpretaciones de las zonas formadas por Cromatografías, así como investigaciones innovadoras fuera y dentro de Brasil. Los resultados presentan investigaciones con la caracterización de tipos de sistemas de gestión y arreglos de producción de diferentes suelos. Además, con el uso de softwares analizar las propiedades de la imagen, distribución y números de partículas para la determinación de grados de tinte, saturación y entropía de color. Y, a través de estadísticas experimentales, la correlación de estos patrones cromatográficos y el contenido mineral y orgánico de los resultados de análisis químicos del suelo. Se concluyó que el avance en las metodologías de comprensión de los resultados de la FCC mostró un crecimiento expresivo en la última década, con el uso de métodos científicos y tecnológicos de alta precisión, abriendo la posibilidad de consolidar el método FCC como Instrumento de análisis de la salud del suelo, así como compuestos. Este artículo se presenta como una lectura para la práctica de nuevas metodologías que conducen a una mayor comprensión de los cromatogramas.

PalabrasClave: Innovación social, salud del suelo, producción ecológica.

\footnotetext{
Abstract

Flat circular chromatography (FCC) is a method of separating components that is divided into two phases, stationary phase and mobile phase. The stationary phase involves the reaction of $1 \%$ Sodium Hydroxide $(\mathrm{NaOH})$ with soil sample and Silver Nitrate impregnation $\left(\mathrm{AgNO}_{3}\right)$ in the filter paper. The mobile phase involves absorbing the filter paper from the Sodium Hydroxide solution with soil. Currently the Plana Circular Chromatography (FCC) indicates a
} 
reading of the life quality of the soil through the harmony of colors and drawings between mineral, organic, energetic and electromagnetic components. This inter-relationship consists of the metabolism of the living soil as well as the availability and efficiency, being the solubility, concentration, constancy and biological quality of the nutrients. In Brazil, the analysis method has been widely disseminated in the agricultural sciences. However, the challenge in interpreting the broad spectrum of chromatogram results was based on the writing of this article whose goal was to accomplisha systematization of the main methodologies of analysis of results and interpretations of the zones formed by chromatograms as well as of innovative searches outside and within Brazil. The results present research with the characterization of types of management and productive arrangements of various soils. Also, with the use of software analyze image properties, distribution and particle numbers for determining tonality degrees, saturation and color entropy. And through experimental statistics the correlation of these chromatographic patterns and mineral and organic content of chemical soil analysis results. It is concluded that the advancement in methodologies of understanding of the FCC's results showed significant growth in the last decade, with the use of high-precision scientific and technological methods, opening the possibility of consolidating the FCC method as a real instrument for soil health

analysis as well as compounds. This article presents itself as a reading for the practice of new methodologies that lead to greater understanding of chromatograms.

Keywords: Social innovation, soil health, organic production.

\section{Introdução}

De fácil assimilação, a Cromatografia Circular Plana (cuja sigla em inglês FCC) compõe alternativa aos métodos de análise convencionais e de difícil acesso. A cromatografia é um instrumento tecnológico acessível a camponeses, técnicos e estudantes que permite acompanhar as transformações e as operações do manejo do sítio agrícola para determinar a "qualidade da saúde dos solos". Uma técnica rápida, de fácil execução e com grande aplicabilidade (PINHEIRO; RESTREPO, 2011).

Ao analisar a qualidade do solo através da cromatografia se faz necessário interpretar imagens geradas, estas chamadas de cromatogramas. A forma subjetiva e auto - interpretativa tem natureza inerente e faz parte do processo de construção desse conhecimento. No entanto a interpretação de um cromatograma requer uma base teórica e experimentada para assim o sujeito, seja ele um pesquisador ou um agricultor, compreender os padrões recorrentes e aparentes nessas imagens e relacioná-las com o estado real desses solos.

Dada a insipiente ciência no meio acadêmico brasileiro, muito embora 80 anos separem os experimentos do cientista Pfeiffer com cromatografia circular plana do presente 
contexto, no Brasil desde sua inserção cerca de 8 anos atrás poucos trabalhos se debruçaram em colaborar com o avanço na compreensão mais profunda das interpretações desses padrões de desenho e imagem.

Realizamos uma sistematização das principais metodologias de análise de resultados e interpretações das zonas formadas por cromatogramas bem como de pesquisas inovadoras. Isso se faz necessário para aprofundar o conhecimento acerca da compreensão, dando maior confiança e acerto as recomendações de manejo, adubação e tratos aos solos ora analisados pelo método FCC.

Investigações foram realizadas no Brasil para a adaptação ao meio e clima tropical da FCC por Pinheiro \& Restrepo (2008). Bons resultados foram obtidos na tradução do comportamento mineralógico, da atividade microbiológica e do manejo de solos através de cromatogramas.

Ribeiro et al. (2016) ao analisar solos amazônicos no P.A. Uatumã, estabeleceu padrões cromatográficos para as condições edafoclimáticas da região e manejo ecológico dos agroecossistemas. Em 18 amostras de solos aplicou a FCC e correlacionou atribuições físicas, como a estrutura do solo, detectando minerais ativos a partir da atividade biológica. A intensidade dessa atividade indica os níveis adequados de nutrientes e o equilíbrio dos mesmos

O método já bastante difundido no meio acadêmico e produtivo brasileiro logra sucesso na aplicação da metodologia adaptada ao nosso clima. Diante do desafio de evoluir os métodos para a interpretação, dando base para a auto - compreensão dos padrões dos cromatogramas, buscamos revisar as pesquisas mais relevantes atualmente.

Com essa pesquisa almejamos colaborar com uma coluna de jovens acadêmicos, pesquisadores e produtores, dando base a partir de experiências promissoras, para a melhor compreensão dos padrões aparentes nas imagens geradas a partir da cromatografia.

\section{Fundamentação Teórica}

Por definição a cromatografia é um método de ação para a separação de componentes dividida em duas fases, uma chamada de fase estacionária e a outra de fase móvel (IUPAC, 1997). As experiências iniciais datam de 1850, contudo sob a condução de Mikail Tswett (1872 - 1919), a cromatografia em 1903 teve seu nascimento de fato considerado. Tswett 
obteve êxito na separação de dois compostos de clorofila (ETTRE \& SAKODYNSKII, 1993; ABRAHAM, 2004).

Atualmente a FCC, juntamente com a cromatografia ascendente de Kolisko, conhecida também como dinamólise capilar ou "Steigbild" (KOLISKO \& KOLISKO, 1978; ZALECKA et al. 2010), compõe um distinto grupo de métodos analíticos, que diferente de Tswett, não buscam a separação de compostos senão utilizam as características dos padrões formados através do processo como indicador de qualidade ou teste de qualidade da amostra. Outros métodos, conhecidos pela formação de imagens (KOKORNACZYK et al., 2011) são citados na literatura como o de cristalização induzida por evaporação via cloreto de cobre (PIVA et al., 1994; BUSSCHER et al., 2010; BAUMGARTNER et al., 2012) e evaporação de gotículas (KOKORNACZYK, et al., 2011; KOKORNACZYK, PARPINELLO, 2014; KOKORNACZYK, TREBBI et al., 2014).

Em todos os métodos citados há a formação de imagens, a complexidade e regularidade desses padrões são consideradas indicadores de qualidade, assim como a formação de padrões irregulares são relacionados a amostras de baixa qualidade (KOKORNACZYK et al., 2012).

É dado ao químico alemão Ehrenfried Pfeiffer (1899 - 1961) a autoria do desenvolvimento da Cromatografia Circular Plana, e sua aplicação, através de papeis filtro, podendo ser realizadas em solos, compostos e culturas (PFEIFFER, 1984).

O método FCC se difere dos métodos de análise convencionais, por meio da reação da solução $\mathrm{NaOH} 1 \%$ com o solo, repica as partículas e substâncias fundamentais que compõe a amostra e imprimi por meio da absorção em papel filtro específico juntamente com uma solução de $\mathrm{AgNO}_{3}$ 0,5\% uma imagem revelada da biodiversidade e sua inter-relação. Para além da quantificação numérica, entra no âmbito da interação entre microrganismos, minerais, proteínas e toda a diversidade que compõe o solo e suas frações (OLIVEIRA et al., 2018) e indica uma leitura da qualidade da vida do solo através da harmonia de cores e desenhos entre os componentes minerais, orgânicos, energéticos e eletromagnéticos. Essa inter-relação compõe o metabolismo do solo vivo assim como a disponibilidade e eficiência, sendo, a solubilidade, concentração, constância e qualidade biológica dos nutrientes (RIBEIRO et al., 2016).

A FCC é aplicável na determinação de qualidade e maturidade de compostos 
orgânicos. Diferentes parâmetros de padrões, larguras e cores das zonas, comprimento dos espigões, contrastes entre as zonas adjacentes e a intensidade de cor das fronteiras se diferem de acordo com a amostra e se correlacionam com os componentes químicos desses compostos (KOKORNACZYK et al., 2016). Em alimentos, a análise de qualidade através da FCC concentra-se na diferenciação de culturas derivadas de diferentes sistemas de manejo em campo. Maeder et al. (2007) detectou diferenças em amostras de trigo cultivadas em experimento a longo prazo. Neste experimento, o FCC foi combinando a outros métodos com formação de imagens e avaliados por um cientista imparcial. Em Knorr (1982) plantas de couve cultivadas sob diferentes condições, especificamente convencional e orgânico, e em diferentes níveis de fertilidade foram analisadas por meio de FCC. Os padrões foram avaliados a partir de um teste de analise sensorial com escala não estruturada, em um painel de 50 alunos. Foram obtidas diferenças significativas entre os dois cultivos e correlações fortes foram encontradas entre os dados sensoriais e os níveis de proteína ou N-N.

Estudos significativos ocorreram na Europa e na região Sulamérica. Além da pesquisa em círculos científicos, o FCC, possui uma metodologia simples, facilmente assimilável por agricultores, estes quase sempre ligados à agricultura orgânica e ou agroecológica. Publicações dão conta de um grupo italiano chamado INTERLAB, associando produtores, cientistas e curiosos, realizam experimentos em laboratórios trocam experiências e se concentram em alguns aspectos da metodologia (INTERLAB, 2011-2015). No Brasil, Pinheiro (2011) publicou o livro “Agronegócios Versus Biopoder camponês” no qual dedica o segundo capítulo a recomendar a aplicação da FCC e as alternativas de reagentes e materiais para uso pelos agricultores.

\section{Metodologia}

As buscas foram realizadas em 4 bases de dados bibliográficas - Periódicos CAPES, PubMED,Cadernos da Agroecologia/ANA, Google Acadêmico e Google Search. Foi considerado para esta pesquisa a não definição de um intervalo de tempo, obviamente observando a pertinência de cada resultado ao objeto da pesquisa. Foram selecionados artigos em língua portuguesa, inglesa e espanhol. Quanto aos termos de pesquisa ou palavras-chave foram utilizados os seguintes: "Cromatografia de Pfeiffer" e "Pfeiffer's Cromatography". Os trabalhos encontrados foram classificados em 4 grupos, dos quais um grupo ligou-se com 
maior qualidade ao objeto da pesquisa, sendo este o objeto da revisão bibliográfica.

\section{Resultados e Discussão}

O estudo da cromatografia circular plana na análise de sustentabilidade dos solos no Brasil foi aprofundado a partir dos estudos de Pinheiro (2011); Pinheiro \& Restrepo (2011) e Restrepo (2014). Esses autores foram a base para outros estudos e aplicações ao longo desses 8 anos. Além disso, a disseminação desta técnica pelo Brasil e outros países sulamericanos se deu a partir de cursos, oficinas, treinamentos, consultorias e palestras preferidas pelo Dr. Sebastião Pinheiro. A tabela 1 apresenta a bibliografia levantada. Foram encontrados 14 trabalhos científicos escritos em língua portuguesa em anais de eventos e repositórios de dissertação e monografias. Mais 5 trabalhos escritos em língua inglesa e outros 3 trabalhos em língua espanhola.

Tabela 1- Bibliografia levantada na revisão de literaturas

\begin{tabular}{|c|c|c|c|c|c|}
\hline Tema & Resumo & Artigo & Monografia & Dissertação & Livro \\
\hline $\begin{array}{l}\text { Aplicabilidade } \\
\text { em sistemas } \\
\text { produtivos }\end{array}$ & $\begin{array}{l}\text { Kehl (2014); } \\
\text { Silva et al. } \\
\text { (2018); } \\
\text { Tenório et al. } \\
\text { (2011). }\end{array}$ & $\begin{array}{l}\text { Campus et al. } \\
\text { (2018); } \\
\text { Saavedra } \\
(2018) .\end{array}$ & & $\begin{array}{l}\text { Bezerra } \\
(2018) .\end{array}$ & $\begin{array}{l}\text { Restrepo } \\
\text { (2014).; }\end{array}$ \\
\hline $\begin{array}{l}\text { Método } \\
\text { (FCC) }\end{array}$ & $\begin{array}{l}\text { Von Wagner } \\
\text { (2013); } \\
\text { Follador } \\
\text { (2016). }\end{array}$ & $\begin{array}{l}\text { Siqueira et al. } \\
\text { (2016) } \\
\text { (AGROECOL) }\end{array}$ & $\begin{array}{l}\text { Novaes et al. } \\
\text { (2017). }\end{array}$ & Pian (2017). & $\begin{array}{l}\text { Pinheiro } \\
\text { (2011); } \\
\text { Restrepo \& } \\
\text { Pinheiro } \\
\text { (2011). }\end{array}$ \\
\hline $\begin{array}{l}\text { (FCC) versus } \\
\text { análise } \\
\text { química }\end{array}$ & & $\begin{array}{l}\text { Perumal et al. } \\
\text { (2016); } \\
\text { Kokornaczyk } \\
\text { et al. }(2016) \text {; }\end{array}$ & $\begin{array}{l}\text { Siqueira } \\
(2016) ; \\
\text { Santana } \\
\text { (2014). }\end{array}$ & & \\
\hline $\begin{array}{l}\text { Métodos de } \\
\text { interpretação } \\
\text { inovadores }\end{array}$ & & $\begin{array}{l}\text { Miranda et al. } \\
\text { (2017); } \\
\text { Ribeiro et al. } \\
\text { (2016). }\end{array}$ & $\begin{array}{l}\text { Graciano } \\
\text { (2018). }\end{array}$ & $\begin{array}{l}\text { Bezerra } \\
(2018) .\end{array}$ & \\
\hline
\end{tabular}

Fonte: Autor

De acordo com a natureza desses trabalhos, foram divididos em 4 temas. $\mathrm{O}$ tema aplicabilidade em sistemas produtivos encontrou os trabalhos de: Campus et al. (2018) que aplicou a Cromatografia de Pfeiffer no acompanhamento de mudanças em solos tratados com biofertilizantes e húmus. Segundo ele, a técnica tem potencial para apresentar as potenciais 
mudanças benéficas na biologia do solo. Para Kehl (2014) concluiu que a técnica da FCC é um instrumento de vital importância para verificar como estão ocorrendo às interações dos constituintes do solo (aeração, minerais, matéria orgânica, proteínas e enzimas) o que não exclui a utilização de outros instrumentos. Silva et al. (2018)afirma a efetividade no uso do método junto com a análise química convencional para um diagnóstico que contemple parâmetros quantitativo e qualitativo. Tenório et al. (2011) conclui que a técnica pode cumprir o papel de autocertificação de alimentos para a garantia de qualidade, segurança e nutrição. Saavedra (2018) aplicou a técnica em solos de Guanajuato, México e concluiu o uso da técnica para extrair características próprias de uma lavoura.

O tema Método (FCC), Pinheiro (2011) apresenta o par e passo da técnica bem como sua aplicação no monitoramento do comportamento enzimático, microbiano do solo e relaciona toda a construção do mundo agrário produtivo com o potencial maléfico causado pelas mudanças climáticas na atualidade. Em Restrepo (2014) a cromatografia de Pfeiffer é um método a mais num portfólio de tecnologias orgânicas de nutrição e defensivos para as plantas. Von Wagner (2013) apresenta uma experiência no uso da técnica e relata o exercício da discussão da metodologia com o contexto vivido pelo químico Pfeiffer e a corrida pela construção da agricultura química ou revolução verde. Siqueira et al. (2016) (AGROECOL) conclui que é notável a falta de escassez de referencias bibliográficas sobre o assunto, e portanto é uma necessidade expor e compartilhar experiências.Novaes et al. (2017) conclui que a técnica é inteiramente utilizável pelo produtor, assim como em outros países como o México, sendo rápida, simples e eficaz. Follador (2016) apresenta cores, formas e padrões e discute as origens da cromatografia. Pian (2017) utilizou a cromatografia para avaliar solos alemães de uma fazenda biodinâmica, destacou o comportamento diferente entre os períodos do ano, e constatou o comportamento mineral e orgânico do solo ser mais forte no inverno e no verão a atividade microbiológica era forte em equilíbrio com os minerais.

Já para a temática (FCC) versus análise química Siqueira (2016) conclui mais uma vez a sua utilização aliada a analise química convencional e capaz de motivar e sensibilizar manejos agroecologicos de agrossistemas. Kokornaczyk et al. (2016) correlacionou padrões cromatográficos com atributos do solo e concluiu fortes correlações entre eles. Perumal et al. (2018) realizou testes em diversos laboratórios convencionais e constatou diferenças significativas para as mesmas amostras de solos. Comparada a FCC com a análise 
convencional e construindo banco de memórias com um conjunto algoritmo construído foi capaz de criar um diagnóstico por imagem com baixa variação.

Por fim o tema Métodos de interpretação inovadores Miranda et al. (2017) utilizou a técnica FCC para monitorar evolução da fertilidade do solo e conclui a eficácia da tecnologia para monitoramento de acompanhamento da sustentabilidade de agroecossistemas. Ribeiro et al. (2016) apresenta aplicação no bioma amazônico, estuda diferentes agroecossistemas e constrói ferramentas de análise quali-quantitativas para diagnósticos da saúde do solo. Bezerra (2018) conclui a que a técnica FCC fornece uma visão geral e confiável do estado do solo. Graciano (2018) correlacionou a FCC a diferentes profundidades do solo e encontrou relações com a zona central dos cromatogramas. Na mesma pesquisa relacionoua ocorrência de enzimas do solo com a zona externa e destacou correlações fortes.

Os resultados serão discutidos conforme as zonas apresentadas nos cromatogramas, para melhor entendimento e compreensão das mesmas, sendo estas: zona central (CZ), zona média (MZ) e zona exterior (EZ).

\section{Zona Central}

Ribeiro et al. (2016) analisando solos Amazônicos a partir da FCC, constitui uma escala de 0 - 100 para a zona central (CZ), quanto a saturação de cor (SC) aplicada a cada um cromatograma. Podemos então constituir dois parâmetros com um intervalo comparativo entre eles: o de baixa saturação de cor (BSC) que significa baixa intensidade ou uma palidez da cor; à alta saturação de cor (ASC) que significa alta intensidade ou uma vivacidade desta cor. Em CZ as cores variantes são: de claro, variando de creme ao branco; ao tom escuro, variando de cinza a preto. Na Figura 01 a pesquisa correlacionou os resultados das 18 amostras com a saturação de cor de cada amostra na escala citada acima BSC/ASC, assim a intensidade de cor foi descrita para o universo das amostras em questão, 18 no total. 
Figura 1 - Escala de SC - saturação de cor para um universo de 18 amostras de solos em Presidente Figueiredo $\mathrm{AM}$

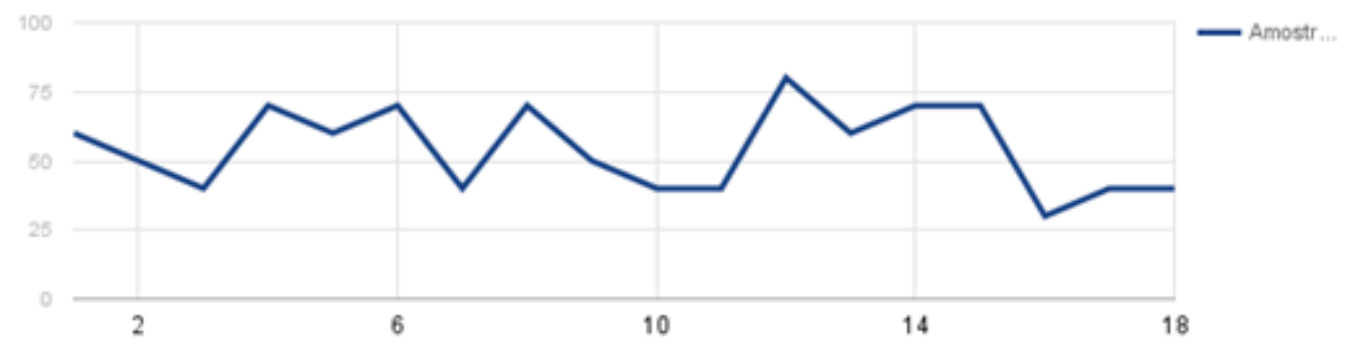

Fonte: Ribeiro et al (2016)

Essa metodologia de interpretação facilita o ordenamento de um determinado universo amostral em análise e possibilita a lúcida compreensão entre as diferenças amostrais.

O método de diferenciação foi subjetivo, ou seja, utilizou-se da observação e sensibilidade na determinação da escala de intensidade. Para iniciar a plotagem do gráfico de SC se faz necessário caracterizar os dois parâmetros base BSC/ASC e a partir deles distribuir as amostras de acordo a afinidade de saturação de cor (SC). Na pesquisa de Ribeiro et. al., (2016) optou-se pelas amostras a seguir na Figura 02 como parâmetros de comparação.

Figura 2 - BSC e ASC para agrupamento de amostras.

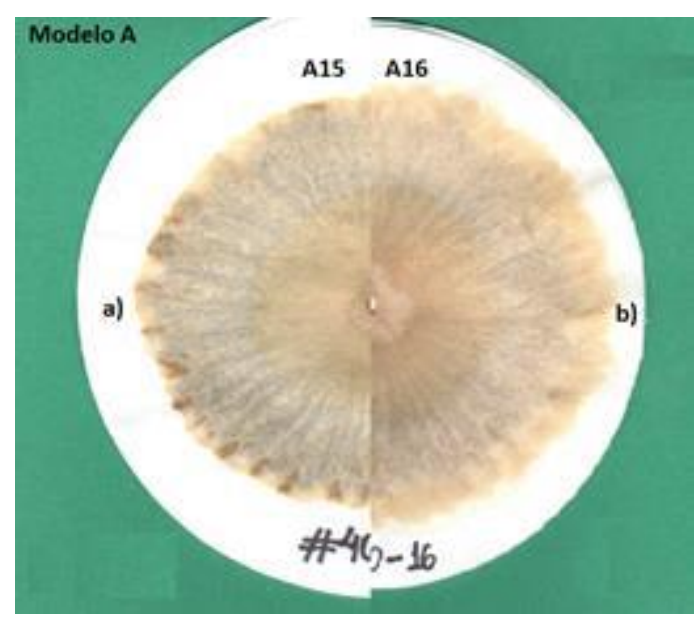

Fonte: Ribeiro et al, (2016)

Para Ribeiro (2016) o grau crescente de intensidade de cor indica a indisponibilidade nutricional, do húmus permanente, da diversidade mineral, da atividade biológica e da integração entre zonas. De acordo com Restrepo (2014) a intensidade da cor varia conforme a 
reação do Hidróxido de Sódio $(\mathrm{NaOH}) 1 \%$ com as seguintes substâncias $\mathrm{N} / \mathrm{NH}_{3} / \mathrm{NO}_{2} / \mathrm{NO}_{3} \mathrm{e}$ as cores variam de acordo com a presença do Oxigênio (ascendência oxidante) e Enxofre (ascendência redutora) estes no momento da coleta.Ribeiro (2016, p. 5) pode estar equivocado na compreensão quanto aos processos que determinam a intensidade de cor, pois além de contradizer Restrepo (2014) o faz também com Kokornaczyk et al. (2016) este último utiliza o parâmetro intensidade para avaliar cromatogramas em pesquisa na Europa.

Quanto à coloração, o "escuro" indica o mínimo metabolismo microbiano aeróbico e máxima fermentação anaeróbica. A cor branca indica a reação do $\mathrm{AgNO}_{3}$ (Nitrato de Prata) com a presença de estruturas nitrogenadas. A cor creme indica a maior plenitude do metabolismo microbiano, enzimático e ação benéfica aos solos (RESTREPO, 2014).

Figura 3 - Gráfico em pizza de 18 amostras de solos em função da área da Zona Central (CZ)

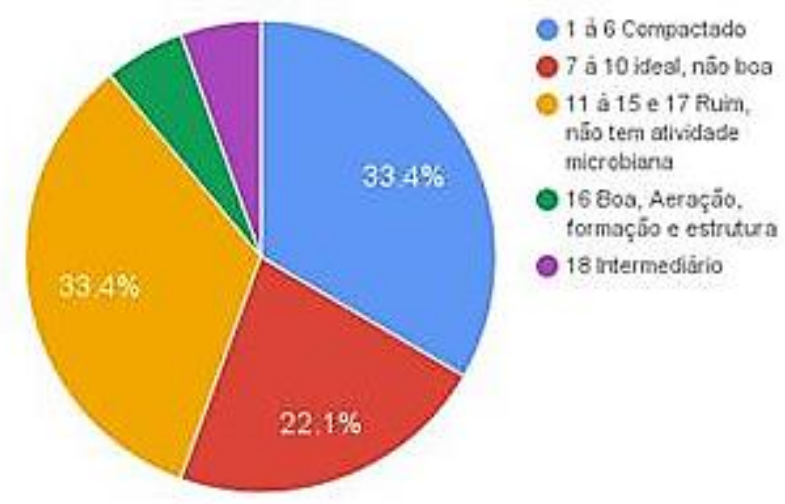

Fonte: Ribeiro et. al. (2016)

Para um conjunto de amostras Ribeiro et al. (2016) apresenta os dados em gráfico em formato de pizza, dividindo o universo amostral qualitativamente. No estudo em questão, o pesquisador encontrou mais de $60 \%$ das amostras em más condições ou péssimas quanto a atividade biológica. Essa metodologia pode ser utilizada para comparações com análises futuras, e desse modo caracterizar em curvas o progresso a atividade biológica, seja de solos ou de compostos orgânicos.

O monitoramento da qualidade de solos manejados agroecologicamente, realizado por 
Miranda et al. (2017) na UR-MECA/UFPB ${ }^{6}$, apontaram o tamanho da área da CZ e sua cor como indicador de qualidade(IQ).

Figura 4 - Progresso da atividade biológica de solos recuperados com manejo agroecológico em URMECA/UFPB numa série em 3 anos de análise, sendo: A - 2015; B - 2016; C - 2017

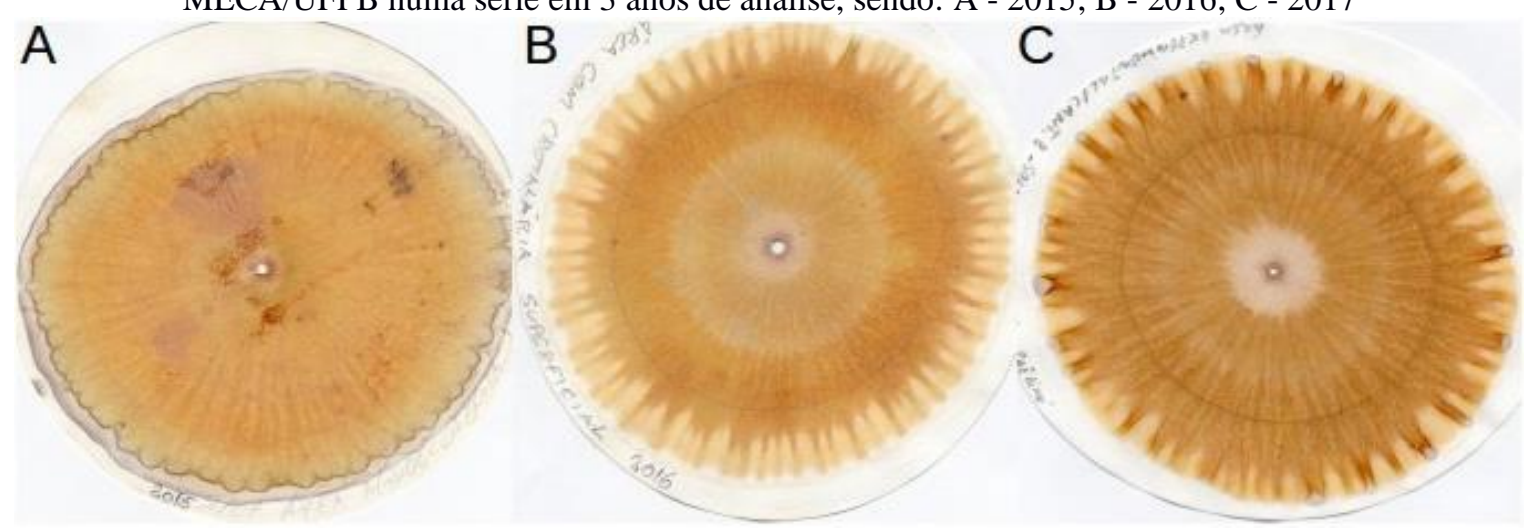

Fonte: Miranda et al. (2017)

Esse estudo apresenta a CZ com uma área específica muito pequena e com coloração escura, pouco se diferenciando da zona posterior. Esses elementos citados não são desejáveis na composição imagética do cromatograma (RESTREPO \& PINHEIRO, 2011). Essa característica indica um solo com pouca estruturação, com dificuldade na troca de gases e o desenvolvimento da biota (Miranda et al., 2017).

Padrões cromatográficos correlacionados com a fertilidade do solo foram encontrados em pesquisa realizada na Europa. Kokornaczyk et al. (2016) correlacionou a avaliação padrão visual cromatográfica com análise computadorizada de imagens (FCC) digitalizadas e com resultados das análises químicas de solos de 16 amostras de solos fornecidas pela fazenda biodinâmica "Le Due Torri” (Spello, Perugia, Itália). As amostras foram coletadas de 10 a 35 cm de profundidade, com manejo convencional e orgânico.

As imagens foram processadas levando em consideração a textura padrão a partir do grau de entropia de cor, através de software ImageJ (COLLINS, 2007) com o analisador de textura plug-in instalado (CABRERA, 2003-2005).

As análises químicas seguiram o regulamento oficial Italiano (MIPAF, 1992-1999) ${ }^{7} \mathrm{e}$

\footnotetext{
${ }^{6}$ A sigla significa Unidade Referência do coletivo MECA (Movimento de Educação do Campo e Agroecologia).

${ }^{7}$ Matéria orgânica (método Wolkley e Black), nitrogênio total (método Kjedahl), troca catiônica, fósforo assimilável, bromo assimilável, cálcio trocável, magnésio trocável, pH, areia, argila e silte.
} 
o conjunto de dados foi analisado por meio de análise de variância (ANOVA) seguida de comparação de média múltipla post hoc com teste de $\operatorname{LSD}^{8}$ usando o software CoStat ${ }^{9}$.

$\mathrm{Na}$ imagem abaixo, aspectos considerados na avaliação padrão visual da pesquisa e 4 cromatogramas a, b, c e d, demonstrando diferentes desenvolvimentos e características dos solos analisados.

Figura 5 - Avaliação padrão visual da pesquisa, Zona Central (CZ), Zona Mediana (MZ), Zona Externa (OZ), raio de CZ, tamanho do Canal (Channel), Espiga (Spike) e Curvas Concêntricas (Concentric Ring).

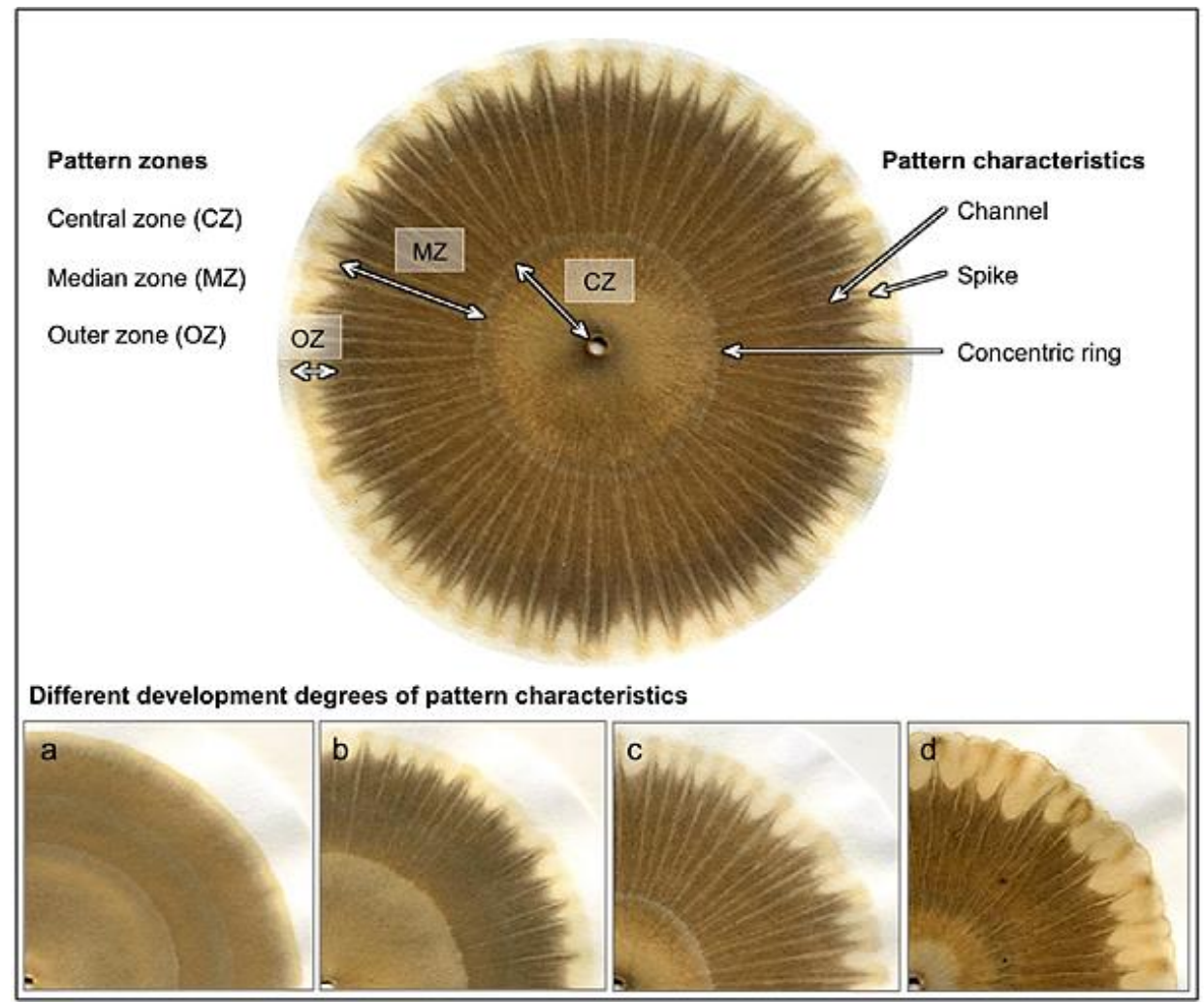

Fonte: Kokornaczyk et al. (2016)

No que tange $\mathrm{CZ}$ a pesquisa encontrou uma correlação marcante inversamente proporcional entre o padrão concêntrico e o padrão radial. Na imagem abaixo, exemplifica o resultado entre a negativa relação entre a ocorrência de raios concêntricos e padrões radiais.

\footnotetext{
${ }^{8}$ LSD - Least Significant Difference.

${ }^{9}$ CoStat v. 6.4, Software CoHort.
} 
Figura 6 -Padrão concêntrico (a) e padrão radial (b). Aspectos apontados no padrão radial: Abertura do canal (Channel's opening), Espiga (Spike), Canal (Channel), Deposito (Deposit), Linha de borda ondulada (Undulated border line) e Remanescentes coloridos (Colored remnant)

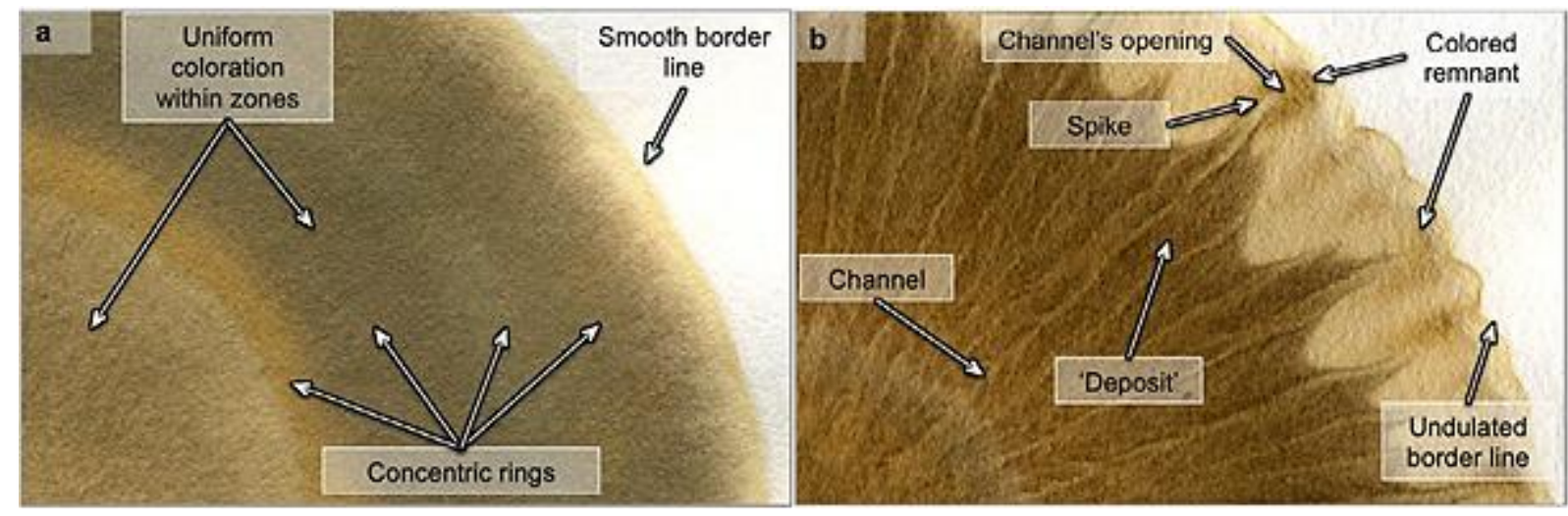

Fonte: Kokornaczyk et al. (2016)

Em padrões concêntricos o raio de $\mathrm{CZ}$ tende ao aumento, assim como a uniformização de cor com outras zonas. Outra tendência encontrada é a turbidez da imagem em correlação positiva com os padrões radiais. Esse resultado é divergente de Ribeiro et al. (2017) que considera a intensidade de cor uma variável com correlação negativa com a fertilidade, ou seja, quando maior for o grau de intensidade, menor será disponibilidade de nutrientes presente no solo.

Kokornaczyk et al. (2016) conclui que: “...a forte diferenciação radial e a coloração intensa dos padrões parecem ser sinais de boa qualidade do solo, enquanto a diferenciação de padrões concêntricos e as cores borradas indicam um solo não fértil...”. No mesmo trabalho, a autora correlaciona à ocorrência dos anéis concêntricos e todas as características dos padrões como, canais, pontas, e intensidade de cor. Para a intensidade de cor encontrou-se uma relação negativa na ordem de r: $-0,93$ com significância de $1 \%(\mathrm{p}<0,0001)$.

Miranda et. al. (2017) corrobora com a autora citada: “... De forma geral, o cromatograma apresenta coloração opaca sem vida, não há integração evidente entre as zonas confirmando, portanto, sua baixa fertilidade e atividade biológica...”.

Graciano et. al. (2018) conclui que na zona central do cromatograma há uma correlação negativa com a resistência à penetração do solo abaixo dos $20 \mathrm{~cm}$ de profundidade, assim como também na média dentro do intervalo $0-40 \mathrm{~cm}$. Isto é, quanto mais compactado estava o solo, menor foi à qualidade da $\mathrm{CZ}$, tendo se confirmado os padrões de qualidade. $\mathrm{E}$ em relação $\mathrm{MZ}$ do cromatograma, que traduz o comportamento mineral do solo apresentou 
uma correlação positiva com o conteúdo orgânico total (COT), além de demonstrar sensibilidade para problemas do solo,estes relacionados á intensidade do revolvimento. Os padrões de qualidade analisados nesta zona dos cromas, com radiações bem marcadas, coloração marrom e integração com as demais zonas, se confirmaram neste estudo.

\section{Zona Média}

A constituição da ZM compreende para alguns autores a Zona Mineral e Zona Protéica (ou Zona da Matéria Orgânica) (PINHEIRO \& RESTREPO, 2008). Abordaremos como uma zona-região integrada e analisaremos de um modo geral os trabalhos recentes que a caracterizam.

Para Ribeiro et al. (2016) é possível constatar nessa zona a presença ou não de minerais. Ao apresentar círculos lineares indica uma membrana inorgânica, portanto sem atividade. As cores podem varia de preto ao alaranjado, menor e maior atividade respectivamente. Nesta zona formam-se os "dentes" segundo o autor e se determina a qualidade de acordo com a harmonia de cores, a evolução radial e harmonia entre as zonas. $\mathrm{Na}$ caracterização dos solos Amazônicos o autor definiu que se as zonas podem ser vistas claramente, ou seja, podem ser diferenciadas facilmente, então define-se que não há harmonia na terra. E quando ocorrer o contrário, as zonas se misturarem e interagirem harmoniosamente pode-se então afirmar que a atividade biológica é adequada.

No trabalho desenvolvido na Itália, uma metodologia interessante foi construída para caracterizar o desenvolvimento da Zona Mediana e assim correlacioná-la com a análise química dessas amostras de solo, assim como com a análise de entropia de cor das imagens digitalizadas. Na Figura 05 ficam visíveis as características sob investigação. Foi realizada a avaliação padrão visual (APV) da Zona Mediana (MZ), contabilizando as características de amplitude da camada, tamanho do Canal (Channel), Espiga (Spike), Concentricidade (Concentric Ring), os depósitos (deposit) e a entropia (textura). Kokornaczyk et al. (2016) atribuiu nessa APV um máximo de 5 pontos para as características, picos e intensidade de cor. Para canais e picos, um ponto foi acreditado quando essas características estavam ausentes e 5 pontos quando o desenvolvimento estava pleno; para a intensidade cor, 1 ponto foi acreditado quando a cor foi visualmente percebida como desfocada, e 5 pontos quando foi sentida como intensa. Pontos intermediários (2, 3 e 4) foram acreditados em casos de desenvolvimento 
característicos intermediário. Para anéis concêntricos característicos (pontuação de 1 a 4 pontos) de acordo com o numero de anéis, um ponto acreditado para cada anel visível no padrão.

Novamente encontrou-se o padrão aplicado a CZ da proporção inversa entre curvas concêntricas e padrões radiais. Padrões concêntricos continham até 4 anéis entre $\mathrm{CZ}$ e $\mathrm{MZ}$. O fator amplitude de MZ não criou nenhuma correlação significativa com outras medidas do padrão. A investigação concluiu que a ocorrência de canais, espigas e depósitos esta diretamente ligada ao padrão radial. Enquanto que a ocorrência menor dessas características está ligada ao padrão concêntrico da imagem.

Graciano et al. (2018) no seu presente estudo apontou que na MZ (zona média) há forte correlação positiva com o carbono da massa microbiana do solo (CBMS), evidenciando a relação desta zona com a biologia do solo. Os padrões que indicam qualidade para esta zona, como terminações em formato de picos irregulares, coloração marrom e proporção ás demais zonas, também se comprovaram neste estudo.

\section{Zona Exterior}

Para Bezerra (2018) a Zona Externa ou, também, pode ser chamada de Zona Enzimática, indica a zona enzimática ou nutricional do cromatograma, valida a grandeza entre a ausência até a ocorrência de uma zona larga e com cores que variam do castanho escuro ao tom ocre ${ }^{10}$. A expressão de boa qualidade são as bolsinhas e nuvens com bordadura em tons café claros e escuros, que mostram o valor biológico e as reservas nutricionais revelando a vitalidade dos solos.

Aplicações realizadas em solos amazônicos por Ribeiro et al. (2016), apresentou padrões que foram classificados em 6 unidades de comportamento (Figura 7) são: baixo intermediário, intermediário, não ideal agulha, não ideal plano, não ideal e ideal.

\footnotetext{
${ }^{10}$ Ocre, é um pigmento terroso que varia entre tons de amarelo dourado e marrom-amarelado claro.
} 
Figura 7 - Padrões de classificação da zona externa (EZ)

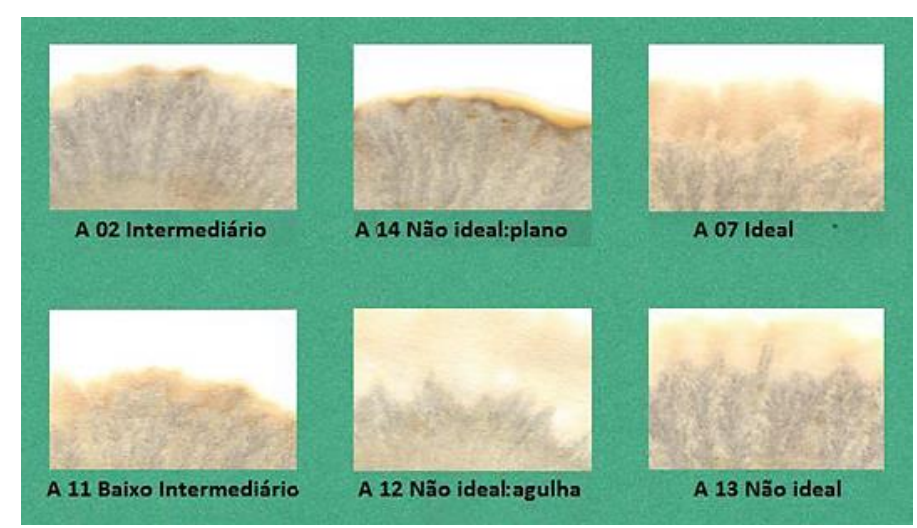

Fonte: Ribeiro et al, (2016)

Observou-se a formação de círculos diminutos chamados de "Dentes de Cavalo" (PINHEIRO, 2011). Variações como, ausência (A12), ocorrência com zona larga com formação de dentes. A amostra A13 demonstra "explosões" e muitas cores com tons de castanho, já a A07 permite a expressão de boa qualidade com bolsas e nuvens provenientes de explosões, bordas de tom café claro ou escuro, o que significa alto valor biológico, reservas nutricionais e integração de zonas. Dentes, grãos, formação de agulhas ou irregulares não são desejáveis, apresentam baixa formação enzimática e baixa variedade nutricional (RIBEIRO et al. 2016).

Para Zona Externa Kokornaczyk et al. (2016) encontraram vários comportamentos correlacionados. De forma negativa entre o raio da zona central e a largura da zona externa. Nesse mesmo estudo, citado exaustivamente em nosso trabalho, a amplitude e entropia de EZ correlacionaram-se negativamente com a ocorrência de padrões concêntricos. Para a ocorrência de padrões radiais, intensidade de cor, canais e picos a largura e entropia de EZ correlacionaram-se positivamente. Quanto aos dados da análise química, conteúdo de matéria orgânica, nitrogênio total, fósforo, bromo e areia criaram correlações positivas com a largura EZ. Contrariando, $\mathrm{pH}$, silte e argila correlacionaram-se negativamente com a largura, canais, picos, intensidade de cor e entropia de EZ.

Graciano et al (2018) apresentou dados de uma expressiva correlação de EZ com as enzimas fosfatase ácida e arilsulfatase, evidenciando sua relação com a atividade enzimática do solo. As expressões das "nuvens enzimáticas" e colorações de tonalidade amarela também puderam ser confirmadas como indicadores de qualidade para esta zona. 


\section{Conclusões}

Conclui-se que os trabalhos realizados nos últimos 10 anos trouxeram importantes avanços para o entendimento e aprofundamento científico da FCC. Faz-se necessário realizar maiores pesquisas correlacionando dados de análise química com dados de avaliação visual. Faz necessário aprofundar pesquisas com a utilização de softwares para a criação de algoritmos de interpretação, que a partir de banco de dados de imagens (cromatogramas), possam facilitar e o entendimento de resultados e recomendações de manejo de agrossistemas. As pesquisas aqui apresentadas trouxeram relevante entendimento de padrões concêntricos e radiais, apontando como importante parâmetro para indicação de qualidade de solos ou mudança de comportamento do mesmo.

\section{Referências}

ABRAHAM, M. H. 100 years of chromatography-or is it 171? Journal of Chromatography A, p. 113-114, 21 out. 2004. Disponível em: $\langle$ http://citeseerx.ist.psu.edu/viewdoc/download?doi=10.1.1.687.8680\&rep=rep1\&type=pdf $>$. Acesso em: 28 maio. 2019.

BEZERRA, L. P. Implantação de Sistemas Agroflorestais na Agricultura Familiar: um caminho para a transição agroecológica. 20 fev. 2018. Disponível em: <https://repositorio.ufscar.br/handle/ufscar/9707>. Acesso em: 31 ago. 2019.

Cabrera J. Texture analyzer [Internet]. c2003-2005; [cited 2015 Dec 9]. Available from: http://rsb.info.nih.gov/ij/plugins/texture.html.

CAMPOS, L. A. F. de et al. Produção de biofertilizante e Análises cromatográficas do solo. Disponível em: <https://2018.febrace.org.br/virtual/2018/AGR/88/>. Acesso em: 31 ago. 2019.

COLLINS, T. J. ImageJ for microscopy. BioTechniques, v. 43, n. 1S, p. S25-S30, 1 jul. 2007. Disponível em: <https://www.future-science.com/doi/10.2144/000112517>. Acesso em: 28 maio. 2019.

ETTRE, L. S.; SAKODYNSKII, K. I. M. S. Tswett and the Discovery of Chromatography I: Early Work (1899-1903). Chromatographia, v. 35, n. 3, p. 223-231, 1 fev. 1993. Disponível em: 〈https://doi.org/10.1007/BF02269707>. Acesso em: 28 maio. 2019.

FOLLADOR, B.; PFEIFFER, E. Portraying Soils and Compost: Color, Form , and Pattern. In: Anais...2015. Disponível em: $<$ https://www.semanticscholar.org/paper/Portraying-Soils-and-Compost-\%3A-Color-\%2CForm-\%2C-and-FolladorPfeiffer/cd814c6593e9173037a11821468a1054d6dc0521\#references>. Acesso em: 31 ago. 2019.

GRACIANO, I. Avaliação da saúde do solo por meio da cromatografia de Pfeiffer: Aspectos metodológicos e aplicações. 2018. Universidade Estadual do Norte do Paraná, 
Bandeirantes (PR), 2018. Disponível em: <https://uenp.edu.br/dissertacao-agronomia/11531igor-graciano/file>. Acesso em: 30 ago. 1989.

INTERLAB [Internet]. c2011-2015; [cited 2015 Dec 9]. Available from: https://sites.google.com/site/sitointerlab/IUPAC. 1997. Compendium of chemical terminology. 2nd ed. Oxford: Blackwell Scientific.

KEHL, L. G. H. SOGLIO, F. K. D. Cromatografia de Pfeiffer como ferramenta para avaliar a qualidade de solo em Sistemas Agroflorestais. XXVI Salão de Iniciação Científica - UFRGS 2014: Conhecimento, formação. Inovação. Porto Alegre (RS).. Disponível em: 〈https://lume.ufrgs.br/handle/10183/114246>. Acesso em: 31 ago. 2019.

KOKORNACZYK, M. O. et al. Self-Organized Crystallization Patterns from Evaporating Droplets of Common Wheat Grain Leakages as a Potential Tool for Quality Analysis. The Scientific World Journal, v. 11, p. 1712-1725, 17 out. 2011. Disponível em: <https://www.ncbi.nlm.nih.gov/pmc/articles/PMC3201687/>. Acesso em: 28 maio. 2019.

KOKORNACZYK, M. O. et al. Qualitative Discrimination between Organic and Biodynamic Sangiovese Red Wines for Authenticity. Analytical Methods, v. 6, n. 18, p. 7484-7488, 22 ago. $2014 . \quad$ Disponível em: <https://pubs.rsc.org/en/content/articlelanding/2014/ay/c4ay00971a>. Acesso em: 28 maio. 2019.

KOKORNACZYK, M. O. et al. Droplet Evaporation Method as a New Potential Approach for Highlighting the Effectiveness of Ultra High Dilutions. Complementary Therapies in Medicine, v. 22, n. 2, p. 333-340, abr. 2014.

KOKORNACZYK, M. O.; DINELLI, G.; BETTI, L. Approximate Bilateral Symmetry in Evaporation-Induced Polycrystalline Structures from Droplets of Wheat Grain Leakages and Fluctuating Asymmetry as Quality Indicator. Die Naturwissenschaften, v. 100, n. 1, p. 111115, jan. 2013.

Kokornacsyk, M. O. Primavera, F. Luneia, R. Baumgartner, S. \& Betti, L. Analysis of soils by means of Pfeiffer's circular chromatography test and comparison to chemical analysis results. In: Biological Agriculture \& Horticulture, an International Jornal for Sustainable Production Systems. ISSN: 0144-8765, 2016.

MIPAF. 1992. Gazzetta Ufficiale della Repubblica Italiana [Official Gazette of the Italian Republic]. Serie Generale n. 121:58-87.

MIPAF. 1999. Gazzetta Ufficiale della Repubblica Italiana [Official Gazette of the Italian Republic]. Serie Generale. 248:1-50.

MIRANDA, A. A. C.; SAllA, L. M. X.; ARAUJO, A. E. de. Uso da Cromatografia de Pfeiffer como indicador de qualidade do solo: monitoramento do manejo agroecológico da UR-MECA/UFPB. Cadernos de Agroecologia, v. 13, n. 1, 22 ago. 2018. Disponível em: $<$ http://cadernos.aba-agroecologia.org.br/index.php/cadernos/article/view/1321>. Acesso em: 31 ago. 2019.

NOVAES, W. de O. F. et al. Estudo da cromatografia de Pfeiffer como alternativa agroecológica para análise de solos. Cadernos de Agroecologia, v. 13, n. 1, 22 ago. 2018. Disponível em: agroecologia.org.br/index.php/cadernos/article/view/1224>. Acesso em: 31 ago. 2019. 
OLIVEIRA, W. S.; OLIVEIRA, M. E. S.; NASCIMENTO, J. T.; coordenadores. Cromatografia de Pfeiffer como teste de qualidade de solos. Apostila da oficina do XI SICOOPES e II FECITIS. Núcleo de Pesquisa em Ciências do Solo e Água da Amazônia NUPECSA do IFPA - Campus Castanhal, 2018.

PERUMAL, K.; ANANTHI, S.; ARUNKUMAR, J. Innovative and Simplest Alternative Analytical Technology (AAT) for Testing Soil Nutrients. JORNAL OF SOIL SCIENCE RESEARCH, v. 1, n. 1, p. 10, 2016. Disponível em: <https://www.tsijournals.com/articles/innovative-and-simplest-alternative-analyticaltechnologyaat-for-testing-soil-nutrients.pdf> .

PFEIFFER, E. Chromatography applied to quality testing. 1984. Wyoming, USA: BioDynamic Farming and Gardening Association:44 p.

PIAN, L. B. Chromatography of Pfeiffer: Principles, Method and Use in Perception of Soils. $\quad$ p. 46, 2017. Disponível <https://www.dottenfelderhof.de/fileadmin/images/landbauschule/projektarbeiten/Projektarbe it_Livia_Pian2016-17_ingles.pdf>. Acesso em: 31 ago. 2019.

Ribeiro, A. V. S.; Nery, I. L. P.; Mariano, J. S.; Maciel, B. C. M.; Nina, N. C. Cromatografia de Pfeiffer aplicada a solos amazônicos no Projeto de Assentamento (P.A.) Uatumã, Presidente Figueiredo - AM. IV Seminário Internacional de Ciência do Ambiente e Sustentabilidade na Amazônia - Amazônia 2030. Manaus, 2016.

SAAVEDRA, T. M.; FIGUEROA, G. A.; CABALLERO, V. P. Chromatography of Pfaiffer in the Analysis of Soils of Productive Systems. Revista Mexicana de Ciencias Agrícolas, v. 9, n. 3, p. 9, 15 maio 2018. Disponível em: <http://www.scielo.org.mx/pdf/remexca/v9n3/2007-0934-remexca-9-03-665-en.pdf >. Acesso em: 31 ago. 2019.

SIQUEIRA, J. B.; MARQUES, G. dos S.; FRANCO, F. S. Construção de Conhecimento Agroecológico Através da Experimentação da Cromatografia de Pfeiffer, uma Análise Qualitativa dos Solos. 16 a 19 de novembro de 2016. AGROECOL 2016. Dourados (MS), Brasil.

SIQUEIRA, I. de. Avaliação da fertilidade e vitalidade do solo pela cromatografia de Pfeiffer e seu potencial para motivar manejos agroecológicos. 18 out. 2016. Disponível em: <http://repositorio.ufsm.br/handle/1/11334>. Acesso em: 31 ago. 2019.

SILVA, P. V. C. et al. Cromatografia de Pfeiffer para avaliação da qualidade do solo em diferentes sistemas de manejo de solos, no Maciço de Baturité. RESUMOS DO VII ENCONTRO DE INICIAÇÃO CIENTÍFICA. V SEMANA UNIVERSITÁRIA, p. 7, 2018. Disponível em: $<$ http://semanauniversitaria.unilab.edu.br/submissao/gerarTrabalho.php?idTrabalho=2378 $>$.

TENÓRIO, L. L.; BENATTO, L.; SANTOS, L. L. C.; COSTA, N. D. F.; COSTA, T. D.; ROCHA, C. T. Cromatografia de Pfeiffer - a autocertificação camponesa. Resumos do VII Congresso Brasileiro de Agroecologia. 12 a 16/12/2011. Fortaleza (CE), Brasil.

VON WAGNER, A. F.; Cromatografia como indicador da saúde do solo. Resumos do VIII Congresso Brasileiro de Agroecologia. 25 a 28 de novembro de 2013. Porto Alegre (RS), Brasil. 